\title{
Correlation between serum albumin and initial GCS in patient with head injury in a tertiary hospital
}

Binit kumar Jha, Mohan Raj Sharma

Department of Surgery, IOM, TUTH, maharajgunj, Kathmandu

Correspondence: Dr Binit kumar Jha, MS Third year resident, Department of Surgery, IOM, TUTH, maharajgunj, Kathmandu

Email: binitjha.95@gmail.com

\begin{abstract}
Introduction: Traumatic brain injury(TBI) is a major cause of disability, death and economic burden to the society. Albumin is a major protein of human. Values fall as a component of metabolic response to injury or infection. Albumin being a negative phase reactant is consumed under states of stress such as TBI.
\end{abstract}

Materials and Methods: This is a descriptive observational study conducted the Department of Surgery ,TUTH over a period of 1 year between 2014 and 2015. Albumin level at admission was correlated with severity of head injury, Glasgow Coma Scale at admission and duration of hospital stay.

Results: Among 59patients with isolated TBI patients from TUTH, 40(68\%) were male and 19(32\%) were female. $55 \%$ of head injuries occurred between the ages of 18-40 years. Out of total patients, fall was the major cause of head injury with $24(41 \%)$ under this category. Nine (15\%) had severe head injury; 22 (37\%) had moderate and $28(47.45 \%)$ patients had severe head injury at admission. Contusion $19(28 \%)$ and subdural hematoma $17(26 \%)$ were the predominant computed tomography findings. Twenty four $(40 \%)$ patients were managed conservatively, thirty five (60\%) patients were operated. The average albumin level at admission for patients with mild, moderate and severe head injury were $41,46 \mathrm{gm} / \mathrm{dl}, 33.76 \mathrm{gm} / \mathrm{dl}, 31.1 \mathrm{gm} / \mathrm{dl}$ respectively. There is a positive correlation between initial serum albumin and GCS at admission ( $p=0.01, R=0.747)$.

Conclusion: There is a positive correlation between initial serum albumin and GCS at admission. Further research on this topic at multiple centres is warranted.

Keywords: albumin, GCS, head injury 